\title{
In vitro Assessment of Antibiotic Resistance Pattern among Lactobacillus Strains Isolated from Goat Milk
}

\author{
Nishchal Thakur, Chetan Sharma, Namita Rokana, Brij Pal Singh, \\ Rohini Devidas Gulhane, Santosh Kumar Mishra, Sunil Kumar Khatkar, \\ Anil Kumar Puniya and Harsh Panwar*
}

\begin{abstract}
Department of Dairy Microbiology, College of Dairy Science and Technology, Guru Angad Dev Veterinary and Animal Sciences University (GADVASU), Ludhiana, Punjab, India
\end{abstract}

\author{
*Corresponding author
}

\section{A B S T R A C T}

This study aimed to assess the antibiotic susceptibility of Lactobacillus strains of goat milk origin. Lactobacilli are generally recognized as safe, but can act as reservoir for genes carrying antibiotic resistance traits; which can further spread to commensal, food spoilage

Keywords

Lactobacillus, Goat milk, Susceptibility, Resistance, Probiotics

Article Info

Accepted:

16 December 2017

Available Online:

10 January 2018 or pathogenic organisms. Lactic acid bacteria (LAB) strains coming from animal sources had significant interaction with antibiotics. Goat milk, being second to cow milk in production and due to its close resemblance to human milk composition, is among favorable source for isolating potential probiotic LAB strains. Herein, this study assessed the antibiotic susceptibility pattern of fourteen Lactobacillus strains isolated from goat milk. The antibiotic susceptibility was recorded against thirty antibiotics following standard disk-diffusion assay. Imipenem, meropenem and nitrofurantoin turned out to be the most effective antibiotics, displaying high zone of inhibition (ZOI) against all the isolates. In contrast, all lactobacilli strains exhibited resistance pattern towards methicillin, oxacillin, cefoxitin, cefmetazole, teicoplanin, vancomycin, ciprofloxacin, ofloxacin, streptomycin, tobramycin, clindamycin and fusidic acid group antibiotics. Overall, GM4 (L. plantarum) displayed highest resistance with clear resistance phenotype against 18/30 antibiotics tested and intermediate resistance against other 5 antibiotics. This study generates new data pertaining to the antibiotic resistance risk of new candidate probiotic strains from goat milk.

\section{Introduction}

Probiotic, prebiotic and synbiotic research is gaining overwhelming response due to their potential health benefits; noteworthy being emerging as a safe and cost-effective alternative to current day antibiotic therapy. Probiotics are mainly dominated by LAB, especially Lactobacillus sp., which constitutes a diverse clade of Gram-positive, catalasenegative, non-spore forming rods or coccobacilli bacteria (Seddik et al., 2017). Probiotics have long history of safe use, are conferred with GRAS (generally recognized as safe) and QPS (Qualified Presumption of Safety) status; however, these strains may act as a reservoir of antibiotic resistance genes that may be transferable. Several reports 
identified genes encoding transferable antibiotic resistance among lactobacilli (Munoz et al., 2014). Therefore, the presence of antibiotic resistance genes in lactobacilli indicates a possible threat to human health. LABs are isolated from several sources including milk, traditional fermented dairy products, faecal matter etc. Strains coming from animal sources viz. milk, fecal matter etc., along with food of animal origin had significant interaction with antibiotics. Antimicrobials are administered to dairy animals for treatment, prophylaxis and growth promotion purpose. Such practices are favorable for genetic selection of resistant strains. In comparison to cattle and buffalo, goats are considered as hardy and adaptive animal and are exposed to antimicrobials to a lower extent. Additionally goat milk is being refereed as a treasure trove, with respect to its nutritional and therapeutic components (Silanikove et al., 2010). Goat milk, due to its close resemblance to human milk oligosaccharide composition (Bode, 2006) is among favorable source for isolating potential probiotic LAB strains. In order to assure the safety of candidate probiotic strains, FAO/WHO guidelines suggest testing these strains for antibiotic resistance patterns. Hence, the present study was undertaken to analyze the status of antibiotic resistance among Lactobacillus isolates of goat milk origin.

\section{Materials and Methods}

\section{Bacterial strains}

This study was attempted to isolate Lactobacillus spp. from raw goat (Beetal) milk obtained from dairy farm of Guru Angad Dev Veterinary and Animal Sciences University (GADVASU) Ludhiana, Punjab, India. Milk samples were processed and lactobacilli were isolated and identified as discussed previously (Panwar et al., 2016;
Sharma et al., 2017a and 2017b). Nucleotide sequence(s) thus obtained were BLAST for similarity search and submitted to NCBI database for Gene Bank accession number (Table 1). Reference Lactobacillus strains viz. Lactobacillus fermentum (NCDC 214), L. plantarum (NCDC 20) and L. rhamnosus (NCDC 19) were procured from National Collection of Dairy Cultures (NCDC), ICARNDRI, Karnal, India.

Reference probiotic strains i.e. L. rhamnosus GG (ATCC 53103) and L. casei (ATCC 393) were procured from American Type Culture Collection (ATCC), USA. All the test strains were maintained and propagated in MRS medium and sub-cultured thrice before antibiotic susceptibility testing.

\section{Test antibiotic disks}

A total of thirty Antibiotic discs belonging to different family were procured from HiMedia Laboratories Pvt. Ltd. Mumbai, India. The list of 26 antibiotics, their dosage and family had been summarized in our previous manuscript (Sharma et al., 2017a); except four new antibiotics namely, Azithromycin (Macrolides), Cefmetazole $\left(2^{\text {nd }}\right.$ generation cephalosporins), Cephalothin ( $1^{\text {st }}$ generation cephalosporins), and Amoxyclav ( $\beta$-lactams).

\section{Antibiotic susceptibility assay}

The susceptibility pattern of different isolates and cultures were evaluated through modified Kirby-Bauer disc-diffusion assay (Sharma et al., 2017a). Isolates with $\mathrm{ZOI} \leq 14 \mathrm{~mm}$ were recorded as resistant (R), $\quad \mathrm{ZOI} \geq 20$ as susceptible (S), and ZOI in between $15-19 \mathrm{~mm}$ were considered as intermediate (I). Antibiotic susceptibility assay was performed in triplicate and the diameters of zone of inhibition were recorded either as resistant $(\mathrm{R})$, sensitive (S) or intermediate (zone diameter $\pm \mathrm{SD}$ ) . 


\section{Results and Discussion}

A total of 14 Lactobacillus strains were identified based on genus specific PCR and $16 \mathrm{~S}$ rRNA sequencing. The identity of isolates along with the NCBI accession numbers has been listed in Table 1. All the isolates were subjected to antibiotic susceptibility testing.

The antibiotic resistance patterns of Lactobacillus strains are displayed in Table 2. Resistance and susceptibility are represented as $\mathrm{R}$ and $\mathrm{S}$ respectively. Intermediate susceptibility has been presented as $\mathrm{ZOI} \pm \mathrm{SD}$ reflecting the inclination towards sensitivity or resistance. All the goat milk lactobacilli displayed sensitivity towards $\beta$-lactams (imipenem, meropenem) and nitrofurantoin. Macrolide (erythromycin) and glycylcycline (tigecycline) group antibiotics also inhibited majority of Lactobacillus sp. isolates, except GM5 and GM10. Ampicilllin, cefotaxime and chloramphenicol were also inhibitory towards most of lactobacilli, except GM4, GM5, GM9 and GM10. A high degree of resistance was recorded against selective $\beta$-lactam (methicillin and oxacillin), glycopeptide (teicoplanin, vancomycin), quinolone (ciprofloxacin, ofloxacin), aminoglycoside (streptomycin, tobramycin), lincosamide (Clindamycin) and fusidane (Fusidic acid) group antibiotics. Among cephalosporins; cefoxitin and cefmetazole were in-effective, while cefuroxime displayed inhibitory activity against few isolates. Ceftazidime inhibited most of the isolates, except GM5. Majority of isolates showed intermediate susceptibility towards tetracycline. A variable resistance/intermediate/sensitive profile was observed against amoxyclav, azithromycin, penicillin, co-trimoxazole, trimethoprim, gentamycin and cephalothin. Overall, GM4 ( $L$. plantarum) showed highest resistance, as 18 out of 30 antibiotics turned out to be ineffective; closely followed by GM8 ( $L$. plantarum) and GM13 (L. plantarum) (17/30).
Similar to Lactobacillus isolates, a varied level of resistance was displayed by reference Lactobacillus (L. plantarum, L. rhamnosus and $L$. fermentum) and probiotic ( $L$. rhamnosus GG and L. casei) strains. All of the reference and probiotic strains were susceptible towards imipenem, ampicilllin, penicillin $\quad(\beta$-lactam $), \quad$ erythromycin (macrolide), cefuroxime, cefotaxime (cephalosporin) and chloramphenicol; whereas displayed resistant pattern towards cefoxitin, cefmetazole (cephalosporins), trimethoprim and co-Trimoxazole. Intermediate to resistance profile was displayed against other antibiotics. Among all the antibiotic groups, cell wall synthesis inhibitors (imipenem, meropenem, ampicilllin and cefotaxime) displayed better inhibitory activity, followed by protein synthesis inhibitors (erythromycin, chloramphenicol and tigecycline).

Our study revealed high resistance of goat milk lactobacilli to tested antibiotics. None of the strain was totally susceptible to all targeted antibiotics and displayed multidrug resistance. All the goat milk lactobacilli were inhibited by imipenem and meropenem, both belonging to carbapenems, the most potent class of $\beta$ lactams family. Our results are in high accordance with Bousmaha-Marroki and Marroki (2015) who also reported high susceptibility of Algerian goat milk lactobacilli towards imipenem. Among other $\beta$-lactams, most of Lactobacillus isolates were sensitive towards ampicillin; sensitive to intermediate against amoxyclav, followed by penicillin; and complete resistance towards methicillin and oxacillin. Our results are in partial concordance with Bousmaha-Marroki and Marroki (2015), who also observed the susceptibility pattern of lactobacilli against penicillin, ampicillin and resistance towards oxacillin. Recent study conducted by Sharma and co-workers (2017a) also observed the resistance pattern of curd and human milk LAB towards methicillin and oxacillin. 
The genetic basis of such resistance might be due to mutations in the penicillin-binding proteins, or due to the presence of genes coding for $\beta$-lactamase (Ashraf and Shah, 2011).

Among cephalosporins, cefotaxime, the $3^{\text {rd }}$ generation cephalosporin was quite effective against majority of goat milk lactobacilli. However, ceftazidime $\left(3^{\text {rd }}\right.$ generation cephalosporin), cefoxitin and cefmetazole $\left(2^{\text {nd }}\right.$ generation cephalosporins) were nearly ineffective. Interestingly, most goat milk lactobacilli displayed intermediate susceptibility towards cephalothin $\left(1^{\text {st }}\right.$ generation cephalosporin). Few other reports also documents high resistance of LAB towards cephalosporins (Gad et al., 2014).

Our results are in agreement to findings of Halder and Shyamapada (2016), who also observed the susceptibility of lactobacilli against cefotaxime. Resistance to cephalosporins can be attributed to the presence of $\beta$-lactamases and/or efflux pumps (Pfeifer et al., 2010), or due to cell wall impermeability (Delgado et al., 2005).

The complete resistance of goat milk Lactobacillus isolates towards glycopeptides (teicoplanin, vancomycin) is in line to other reports (Nawaz et al., 2011). Intrinsic resistance towards vancomycin might be due to the presence of D-Ala-D-lactate in the cell wall peptidoglycan, rather than the D-ala-Dala dipeptide moiety. Vancomycin resistance is chromosomally encoded and nontransmissible (Marroki et al., 2011).

All goat milk lactobacilli exhibited strong resistance pattern towards quinolones (ciprofloxacin and ofloxacin). Resistance towards quinolones may be explained with the nucleotide substitutions (gyrA and/or gyrB) within the quinolone-resistance determining region of DNA gyrase subunits (Baines and Wilcox, 2015), or due to mutation in topoisomerase IV (Hummel et al., 2007).

Table.1 List of goat milk LAB strains used in this study. IPhp denotes lab code and GM (goat milk) signifies isolate number

\begin{tabular}{|l|l|l|}
\hline Lab code & Identity & \multicolumn{1}{|l}{$\begin{array}{l}\text { NCBI Accession } \\
\text { numbers }\end{array}$} \\
\hline IPhp-GM4 & Lactobacillus plantarum & KX943025 \\
\hline IPhp-GM5 & L. rhamnosus & KX943026 \\
\hline IPhp-GM6 & L. plantarum & KX943027 \\
\hline IPhp-GM17 & L. plantarum & KX943028 \\
\hline IPhp-GM18 & L. plantarum & KX943029 \\
\hline IPhp-GM19 & L. pentosus & KY658473 \\
\hline IPhp-GM10 & L. plantarum & KX943030 \\
\hline IPhp-GM11 & L. plantarum & KY658474 \\
\hline IPhp-GM12 & L. plantarum & KY658475 \\
\hline IPhp-GM13 & L. plantarum & KY658477 \\
\hline IPhp-GM14 & L. plantarum & KY658478 \\
\hline IPhp-GM15 & L. plantarum & KY658479 \\
\hline IPhp-GM17 & L. fermentum & KY658480 \\
\hline IPhp-GM18 & L. gasseri & \\
\hline
\end{tabular}


Table.2 Antibacterial susceptibility profile of goat milk lactobacilli, standard Lactobacillus and reference probiotic strains towards commercially used antibiotics

\begin{tabular}{|c|c|c|c|c|c|c|c|c|c|c|c|c|c|c|c|c|c|c|c|c|}
\hline \multirow[t]{2}{*}{$\begin{array}{l}\text { Antibacterial } \\
\text { drugs }\end{array}$} & \multirow{2}{*}{$\begin{array}{l}\text { Disc code } \\
\text { with } \\
\text { concen- } \\
\text { tration } \\
\text { (mcg) }\end{array}$} & \multicolumn{14}{|c|}{ Goat milk (GM) isolates } & \multicolumn{3}{|c|}{$\begin{array}{c}\text { Standard } \\
\text { Lactobacillus sp. }\end{array}$} & \multicolumn{2}{|c|}{$\begin{array}{l}\text { Probiotic } \\
\text { strains }\end{array}$} \\
\hline & & $\begin{array}{l}\text { GM } \\
4\end{array}$ & GM5 & GM6 & GM7 & $\begin{array}{l}\text { G } \\
\text { M8 }\end{array}$ & GM9 & $\begin{array}{l}\text { GM } \\
10\end{array}$ & $\begin{array}{l}\text { GM } \\
11\end{array}$ & $\begin{array}{l}\mathrm{G} \\
\mathrm{M} \\
12\end{array}$ & $\begin{array}{l}\text { GM } \\
13\end{array}$ & $\begin{array}{l}\text { GM } \\
14\end{array}$ & $\begin{array}{l}\mathrm{G} \\
\mathrm{M} \\
15\end{array}$ & $\begin{array}{l}\mathrm{G} \\
\mathrm{M} \\
17\end{array}$ & $\begin{array}{l}\mathrm{G} \\
\mathrm{M} \\
18\end{array}$ & 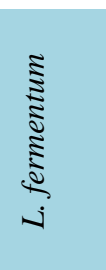 & 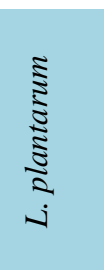 & 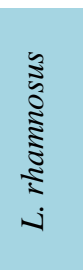 & $\begin{array}{l}0 \\
0 \\
\vdots \\
\vdots \\
\vdots \\
\vdots \\
\vdots \\
\vdots \\
\vdots \\
\text { j }\end{array}$ & $\begin{array}{l}\tilde{\delta} \\
\tilde{3} \\
\text { u }\end{array}$ \\
\hline Ampicillin & $\operatorname{AMP}(10)$ & $\begin{array}{l}18 \pm \\
0.57\end{array}$ & $\begin{array}{l}17 \\
\pm 0\end{array}$ & S & S & $\mathrm{S}$ & $\mathrm{S}$ & $\mathrm{S}$ & $\mathrm{S}$ & S & $\mathrm{S}$ & $\mathrm{S}$ & $\mathrm{S}$ & $\mathrm{S}$ & $\mathrm{S}$ & S & $\mathrm{S}$ & $\mathrm{S}$ & S & $\mathrm{S}$ \\
\hline Imipenem & $\begin{array}{l}\text { IMP } \\
(10)\end{array}$ & $\mathrm{S}$ & $\mathrm{S}$ & S & S & S & $\mathrm{S}$ & S & S & S & S & S & S & $\mathrm{S}$ & $\mathrm{S}$ & S & $\mathrm{S}$ & S & S & $\mathrm{S}$ \\
\hline Meropenem & MRP (10) & S & $\mathrm{S}$ & $\mathrm{S}$ & $\mathrm{S}$ & $\mathrm{S}$ & $\mathrm{S}$ & $\mathrm{S}$ & $\mathrm{S}$ & $\mathrm{S}$ & $\mathrm{S}$ & $\mathrm{S}$ & $\mathrm{S}$ & $\mathrm{S}$ & $\mathrm{S}$ & $\mathrm{S}$ & $\mathrm{S}$ & $\mathrm{R}$ & S & $\mathrm{S}$ \\
\hline Methicillin & MET (5) & $\mathrm{R}$ & $\mathrm{R}$ & $\mathrm{R}$ & $\mathrm{R}$ & $\mathrm{R}$ & $\mathrm{R}$ & $\mathrm{R}$ & $\mathrm{R}$ & $\mathrm{R}$ & $\mathrm{R}$ & $\mathrm{R}$ & $\mathrm{R}$ & $\mathrm{R}$ & $\mathrm{R}$ & $\mathrm{R}$ & $\begin{array}{c}17 \pm 0 \\
57\end{array}$ & $\mathrm{R}$ & $\mathrm{R}$ & $\mathrm{R}$ \\
\hline Oxacillin & OX (1) & $\mathrm{R}$ & $\mathrm{R}$ & $\mathrm{R}$ & $\mathrm{R}$ & $\mathrm{R}$ & $\mathrm{R}$ & $\mathrm{R}$ & $\mathrm{R}$ & $\mathrm{R}$ & $\mathrm{R}$ & $\mathrm{R}$ & $\mathrm{R}$ & $\mathrm{R}$ & $\mathrm{R}$ & $\begin{array}{l}19 \pm 0 \\
57\end{array}$ & $\begin{array}{c}19 \pm 0 \\
5\end{array}$ & $\mathrm{R}$ & $\mathrm{R}$ & $\mathrm{R}$ \\
\hline Penicillin & $\mathrm{P}(10)^{*}$ & $\mathrm{R}$ & $\mathrm{S}$ & $16 \pm 1$ & $18 \pm 0$ & $\begin{array}{c}16 \pm \\
0.5 \\
7\end{array}$ & $\begin{array}{l}17 \pm \\
0.57\end{array}$ & $18 \pm 1$ & S & S & $\mathrm{S}$ & S & $\begin{array}{c}17 \\
\pm \\
0.5 \\
7\end{array}$ & $\mathrm{~S}$ & $\mathrm{~S}$ & $\mathrm{~S}$ & $\mathrm{~S}$ & S & S & S \\
\hline Cefuroxime & AMC (30) & $\mathrm{R}$ & $\mathrm{S}$ & $\mathrm{R}$ & $\begin{array}{l}16 \pm \\
0.57\end{array}$ & $\mathrm{R}$ & $\mathrm{R}$ & $\mathrm{R}$ & $\mathrm{R}$ & $\mathrm{R}$ & $\mathrm{R}$ & $\mathrm{R}$ & $\mathrm{R}$ & $\mathrm{R}$ & $\mathrm{R}$ & S & $\mathrm{S}$ & $\mathrm{S}$ & $\mathrm{S}$ & $\begin{array}{l}19 \\
\pm 0\end{array}$ \\
\hline Cefoxitin & CEP (30) & $\mathrm{R}$ & $\mathrm{R}$ & $\mathrm{R}$ & $\mathrm{R}$ & $\mathrm{R}$ & $\mathrm{R}$ & $\mathrm{R}$ & $\mathrm{R}$ & $\mathrm{R}$ & $\mathrm{R}$ & $\mathrm{R}$ & $\mathrm{R}$ & $\mathrm{R}$ & $\mathrm{R}$ & $\mathrm{R}$ & $\mathrm{R}$ & $\mathrm{R}$ & $\mathrm{R}$ & $\mathrm{R}$ \\
\hline Ceftazidime & CXM (30) & $\mathrm{R}$ & $\mathrm{S}$ & $\mathrm{R}$ & $\mathrm{R}$ & $\mathrm{R}$ & $\mathrm{R}$ & $\mathrm{R}$ & $\mathrm{R}$ & $\mathrm{R}$ & $\mathrm{R}$ & $\mathrm{R}$ & $\mathrm{R}$ & $\mathrm{R}$ & $\mathrm{R}$ & $\begin{array}{l}18 \pm 0 \\
57\end{array}$ & $17 \pm 0$ & $\mathrm{R}$ & $\mathrm{R}$ & $\mathrm{S}$ \\
\hline Cefotaxime & CX (30) & $\begin{array}{l}18 \pm \\
0.57\end{array}$ & $\mathrm{~S}$ & S & S & $\mathrm{S}$ & $\mathrm{S}$ & $\begin{array}{l}16 \pm \\
0.57\end{array}$ & S & $\mathrm{S}$ & S & $\mathrm{S}$ & $\mathrm{S}$ & $\mathrm{S}$ & $\mathrm{S}$ & S & S & S & S & S \\
\hline Teicoplanin & CMZ (30) & $\mathrm{R}$ & $\mathrm{R}$ & $\mathrm{R}$ & $\mathrm{R}$ & $\mathrm{R}$ & $\mathrm{R}$ & $\mathrm{R}$ & $\mathrm{R}$ & $\mathrm{R}$ & $\mathrm{R}$ & $\mathrm{R}$ & $\mathrm{R}$ & $\mathrm{R}$ & $\mathrm{R}$ & $\begin{array}{c}16 \pm 0 \\
57\end{array}$ & $\mathrm{R}$ & $\mathrm{R}$ & $\mathrm{R}$ & $\begin{array}{l}16 \\
\pm 0\end{array}$ \\
\hline Vancomycin & CAZ (30) & $\mathrm{R}$ & $\mathrm{R}$ & $\mathrm{R}$ & $\mathrm{R}$ & $\mathrm{R}$ & $\mathrm{R}$ & $\mathrm{R}$ & $\mathrm{R}$ & $\mathrm{R}$ & $\mathrm{R}$ & $\mathrm{R}$ & $\mathrm{R}$ & $\mathrm{R}$ & $\mathrm{R}$ & $\begin{array}{c}15 \pm 0 \\
57\end{array}$ & $\mathrm{R}$ & $\mathrm{R}$ & $\mathrm{R}$ & $\mathrm{R}$ \\
\hline Ciprofloxacin & CTX (30) & $\mathrm{R}$ & $\mathrm{R}$ & $\mathrm{R}$ & $\mathrm{R}$ & $\mathrm{R}$ & $\mathrm{R}$ & $\mathrm{R}$ & $\mathrm{R}$ & $\mathrm{R}$ & $\mathrm{R}$ & $\mathrm{R}$ & $\mathrm{R}$ & $\mathrm{R}$ & $\mathrm{R}$ & $\mathrm{S}$ & $\begin{array}{c}18 \pm 0 \text {. } \\
57\end{array}$ & $\mathrm{R}$ & $16 \pm 0$ & $\mathrm{R}$ \\
\hline Ofloxacin & TEI (30) & $\mathrm{R}$ & $\mathrm{R}$ & $\mathrm{R}$ & $\mathrm{R}$ & $\mathrm{R}$ & $\mathrm{R}$ & $\mathrm{R}$ & $\mathrm{R}$ & $\mathrm{R}$ & $\mathrm{R}$ & $\mathrm{R}$ & $\mathrm{R}$ & $\mathrm{R}$ & $\mathrm{R}$ & $\mathrm{R}$ & $16 \pm 1$ & $\mathrm{R}$ & $\mathrm{R}$ & $16 \pm 0$ \\
\hline Gentamicin & VA (30) & $\mathrm{R}$ & $15 \pm$ & $16 \pm 1$ & $15 \pm$ & $\mathrm{R}$ & $\mathrm{R}$ & $15 \pm$ & $15 \pm 0$ & $\mathrm{R}$ & $\mathrm{R}$ & $\mathrm{R}$ & $\mathrm{R}$ & $\mathrm{R}$ & $\mathrm{R}$ & $\mathrm{S}$ & $16 \pm$ & 15 & $\mathrm{~S}$ & $\mathrm{~S}$ \\
\hline
\end{tabular}


Int.J.Curr.Microbiol.App.Sci (2018) 7(1): 2108-2116

\begin{tabular}{|c|c|c|c|c|c|c|c|c|c|c|c|c|c|c|c|c|c|c|c|c|}
\hline & & & 0.57 & & 0.57 & & & 0.57 & & & & & & & & & 0.57 & & & \\
\hline Streptomycin & CIP (5) & $\mathrm{R}$ & $\mathrm{R}$ & $\mathrm{R}$ & $\mathrm{R}$ & $\mathrm{R}$ & $\mathrm{R}$ & $\mathrm{R}$ & $\mathrm{R}$ & $\mathrm{R}$ & $\mathrm{R}$ & $\mathrm{R}$ & $\mathrm{R}$ & $\mathrm{R}$ & $\mathrm{R}$ & $\mathrm{R}$ & $\begin{array}{l}15 \pm \\
0.57\end{array}$ & $\mathrm{R}$ & S & $15 \pm 0$ \\
\hline Tobramycin & $\mathrm{OF}(5)$ & $\mathrm{R}$ & $\mathrm{R}$ & $\mathrm{R}$ & $\mathrm{R}$ & $\mathrm{R}$ & $\mathrm{R}$ & $\mathrm{R}$ & $\mathrm{R}$ & $\mathrm{R}$ & $\mathrm{R}$ & $\mathrm{R}$ & $\mathrm{R}$ & $\mathrm{R}$ & $\mathrm{R}$ & $\begin{array}{l}16 \pm \\
0.57\end{array}$ & $\mathrm{R}$ & $\mathrm{R}$ & $\mathrm{R}$ & $\mathrm{R}$ \\
\hline $\begin{array}{l}\text { Chloramphen } \\
\text { icol }\end{array}$ & GEN (10) & S & $\mathrm{R}$ & S & S & S & $\begin{array}{l}19 \pm \\
0.57\end{array}$ & S & S & S & S & S & S & S & S & S & S & S & $19 \pm 0$ & $16 \pm 1$ \\
\hline Clindamycin & S (300) & $\mathrm{R}$ & $\mathrm{R}$ & $\mathrm{R}$ & $\mathrm{R}$ & $\mathrm{R}$ & $\mathrm{R}$ & $\mathrm{R}$ & $\mathrm{R}$ & $\mathrm{R}$ & $\mathrm{R}$ & $\mathrm{R}$ & $\mathrm{R}$ & $\mathrm{R}$ & $\mathrm{R}$ & S & 19 & $\mathrm{~S}$ & S & $\begin{array}{l}15 \pm \\
0.57\end{array}$ \\
\hline Erythromycin & TOB (10) & $\mathrm{S}$ & $\mathrm{R}$ & $\mathrm{S}$ & S & S & S & S & $\mathrm{S}$ & $\mathrm{S}$ & $\mathrm{S}$ & $\mathrm{S}$ & S & $\mathrm{S}$ & $\mathrm{S}$ & S & S & $\mathrm{S}$ & $\mathrm{S}$ & S \\
\hline Fusidic acid & $\mathrm{CD}(2)$ & $\mathrm{R}$ & $\mathrm{R}$ & $\mathrm{R}$ & $\mathrm{R}$ & $\mathrm{R}$ & $\mathrm{R}$ & $\mathrm{R}$ & $\mathrm{R}$ & $\mathrm{R}$ & $\mathrm{R}$ & $\mathrm{R}$ & $\mathrm{R}$ & $\mathrm{R}$ & $\mathrm{R}$ & $S$ & $\mathrm{R}$ & $16 \pm 0$ & $\mathrm{R}$ & $\mathrm{R}$ \\
\hline $\begin{array}{l}\text { Nitrofurantoi } \\
\mathrm{n}\end{array}$ & E (15) & $\mathrm{S}$ & S & $\mathrm{S}$ & S & $\mathrm{S}$ & S & $\mathrm{S}$ & $\mathrm{S}$ & $\mathrm{S}$ & $\mathrm{S}$ & S & $\mathrm{S}$ & $\mathrm{S}$ & $\mathrm{S}$ & S & S & $18 \pm 0$ & $\mathrm{~S}$ & $\begin{array}{c}19 \pm 0 . \\
57\end{array}$ \\
\hline Tetracycline & AZM (15) & $\begin{array}{l}17 \pm \\
0.57\end{array}$ & S & $18 \pm 1$ & $\begin{array}{l}15 \pm \\
0.57\end{array}$ & $\begin{array}{c}15 \pm \\
0.5 \\
7\end{array}$ & $18 \pm 0$ & $16 \pm 1$ & $\begin{array}{l}18 \pm \\
0.57\end{array}$ & $\begin{array}{c}17 \\
\pm \\
0.5 \\
7\end{array}$ & $15 \pm 0$ & $17 \pm 1$ & $\begin{array}{c}16 \\
\pm \\
0.5 \\
7\end{array}$ & $\begin{array}{l}19 \\
\pm 0\end{array}$ & $\begin{array}{c}18 \\
\pm \\
0.5 \\
7\end{array}$ & S & S & $\begin{array}{l}16 \pm \\
0.57\end{array}$ & $\mathrm{~S}$ & $16 \pm 0$ \\
\hline Tigecycline & $\mathrm{FC} \mathrm{(10)}$ & S & S & $\mathrm{S}$ & S & $\mathrm{S}$ & $\mathrm{S}$ & $\begin{array}{l}19 \pm \\
0.57\end{array}$ & $\mathrm{~S}$ & $\mathrm{~S}$ & $\mathrm{~S}$ & S & $\mathrm{S}$ & $\mathrm{S}$ & S & S & $\begin{array}{c}19 \pm 0 \\
57\end{array}$ & S & S & $15 \pm 1$ \\
\hline $\begin{array}{l}\text { Co- } \\
\text { Trimoxazole }\end{array}$ & TGC (15) & $\mathrm{R}$ & $\mathrm{R}$ & $\mathrm{R}$ & $\mathrm{R}$ & $\mathrm{R}$ & $\begin{array}{l}15 \pm \\
0.57\end{array}$ & $\mathrm{R}$ & $\begin{array}{l}17 \pm \\
0.57\end{array}$ & $\begin{array}{l}19 \\
\pm 0\end{array}$ & $\begin{array}{l}16 \pm \\
0.57\end{array}$ & $\mathrm{R}$ & $\begin{array}{l}15 \\
\pm 1\end{array}$ & $\begin{array}{l}17 \\
\pm 0\end{array}$ & $\begin{array}{c}15 \\
\pm \\
0.5 \\
7\end{array}$ & $\mathrm{R}$ & $\mathrm{R}$ & $\mathrm{R}$ & $\mathrm{R}$ & $\begin{array}{c}17 \pm 0 \\
57\end{array}$ \\
\hline Trimethoprim & COT (25) & $\mathrm{R}$ & $\mathrm{R}$ & $\mathrm{R}$ & $\mathrm{R}$ & $\mathrm{R}$ & $\begin{array}{l}16 \pm \\
0.57\end{array}$ & $\mathrm{R}$ & $16 \pm 0$ & $\begin{array}{c}18 \\
\pm \\
0.5 \\
7\end{array}$ & $\mathrm{R}$ & $\begin{array}{l}15 \pm \\
0.57\end{array}$ & $\begin{array}{l}16 \\
\pm 1\end{array}$ & $\begin{array}{c}15 \\
\pm \\
0.5 \\
7\end{array}$ & $\mathrm{R}$ & $\mathrm{R}$ & $\mathrm{R}$ & $\mathrm{R}$ & $\mathrm{R}$ & $\mathrm{R}$ \\
\hline Azithromycin & TR (5) & $\begin{array}{l}18 \pm \\
0.57\end{array}$ & $S$ & $18 \pm 0$ & $\begin{array}{l}19 \pm \\
0.57\end{array}$ & $\begin{array}{c}19 \pm \\
0\end{array}$ & $18 \pm 0$ & $\begin{array}{c}17 \pm \\
1\end{array}$ & $\begin{array}{l}18 \pm \\
0.57\end{array}$ & $\mathrm{~S}$ & $\begin{array}{l}18 \pm \\
0.57\end{array}$ & $\begin{array}{l}19 \pm \\
0.57\end{array}$ & $S$ & $\begin{array}{l}19 \\
\pm 0\end{array}$ & $\mathrm{~S}$ & $S$ & 19 & $\begin{array}{c}17 \pm 0 \\
57\end{array}$ & $18 \pm 0$ & $\mathrm{~S}$ \\
\hline Cefmetazole & C (30) & $\mathrm{R}$ & $\mathrm{R}$ & $\mathrm{R}$ & $\mathrm{R}$ & $\mathrm{R}$ & $\mathrm{R}$ & $\mathrm{R}$ & $\mathrm{R}$ & $\mathrm{R}$ & $\mathrm{R}$ & $\mathrm{R}$ & $\mathrm{R}$ & $\mathrm{R}$ & $\mathrm{R}$ & $\mathrm{R}$ & $\mathrm{R}$ & $\mathrm{R}$ & $\mathrm{R}$ & $\mathrm{R}$ \\
\hline Cephalothin & NIT (300) & $\begin{array}{l}17 \pm \\
0.57\end{array}$ & $\begin{array}{l}19 \pm \\
0.57\end{array}$ & $16 \pm 1$ & $\begin{array}{l}16 \pm \\
0.57\end{array}$ & $\begin{array}{c}15 \pm \\
0\end{array}$ & $\mathrm{R}$ & $\begin{array}{l}16 \pm \\
0.57\end{array}$ & $\begin{array}{l}17 \pm \\
0.57\end{array}$ & $\begin{array}{l}18 \\
\pm 0\end{array}$ & $\mathrm{R}$ & $16 \mathrm{I}$ & $\begin{array}{c}19 \\
\pm \\
0.5 \\
7\end{array}$ & $\mathrm{R}$ & $\begin{array}{l}19 \\
\pm 0\end{array}$ & $18 \pm 0$ & $\begin{array}{c}16 \pm 0 \\
57\end{array}$ & $15 \pm 1$ & $\begin{array}{c}16 \pm 0 \\
57\end{array}$ & $19 \pm 0$ \\
\hline Amoxyclav & TE (30) & S & S & S & $\begin{array}{l}18 \pm \\
0.57\end{array}$ & $\begin{array}{c}19 \pm \\
0\end{array}$ & S & $\begin{array}{l}16 \pm \\
0.57\end{array}$ & S & $\begin{array}{l}16 \\
\pm 1\end{array}$ & $\begin{array}{l}17 \pm \\
0.57\end{array}$ & $\begin{array}{l}18 \pm \\
0.57\end{array}$ & $\mathrm{~S}$ & S & $\mathrm{S}$ & S & S & $\begin{array}{c}18 \pm 0 \\
57\end{array}$ & $19 \pm 0$ & S \\
\hline
\end{tabular}


In our study different degree of sensitivity to resistance pattern has been observed against protein synthesis inhibitors. Previously, it has been documented that lactobacilli are generally resistant to aminoglycosides and susceptible to other protein synthesis inhibitors such as, chloramphenicol, macrolides and tetracycline (Karapetkov et al., 2011). In agreement to above publish reports, we have also noted down the high resistance pattern against aminoglycosides (streptomycin, tobramycin), with an exception of gentamicin, against which both resistance and intermediate susceptibility was recorded. Intermediate susceptibility to resistance pattern of lactobacilli against gentamicin has also been reported earlier (Jiang et al., 2016). Among LAB, resistance to aminoglycosides is mainly due to enzymatic inactivation by aminoglycoside modifying enzymes (AMEs). Three main AMEs i.e. N-acetyl-transferases (AACs), O-phospho-transferases (APHs) and O-nucleotidyl-transferases (ANTs) confers resistance towards aminoglycoside. In this study, majority of Lactobacillus isolates displayed susceptibility towards erythromycin and intermediate to susceptible pattern towards azithromycin. These findings can be substantiated with earlier reports, wherein LAB strains showed susceptibility towards erythromycin and azithromycin (Bhuiyan et al., 2017).

Among other protein synthesis inhibitors explored in this study, lactobacilli displayed remarkable sensitivity towards nitrofurantoin (a synthetic antimicrobial derived from furan by the addition of a nitro group and side chain containing hydantoin). On the other hand, a high resistance to clindamycin and fusidic acid was observed. Our results are in concurrence with other reports, where lactobacilli displayed susceptibility to nitrofurantoin (Bouridane et al., 2016). Similar to this study, Goldstein et al., (2015) also reported sensitivity of lactobacilli towards tigecycline and chloramphenicol. In contrary to our results, Bhuiyan et al., (2017) documented resistance pattern of goat milk lactobacilli towards tetracycline. In compliance to our findings, previous studies also revealed that lactobacilli were resistant to clindamycin (Ashraf and Shah, 2011) as well as to fusidic acid (Jose et al., 2015).

Further, most of the goat milk lactobacilli displayed intermediate to resistance phenotype towards folic acid synthesis inhibitors (co-trimoxazole and trimethoprim). These findings are supported by Anas et al., (2014) who also observed that goat milk $L$. plantarum displayed intermediate resistance to co-trimoxazole. Bousmaha-Marroki and Marroki (2015) reported complete resistance of Algerian goat's milk lactobacilli against trimethoprim. Presence of single nucleotide polymorphisms could be responsible for resistance towards trimethoprim (van Hoek et al., 2011).

Overall, goat milk lactobacilli displayed a diverse level of sensitivity/resistance pattern. Therefore, Lactobacillus strains depicting antibiotic resistance pattern could be explored in restoring the gut microbiota during and after antibiotic treatment. This assumption is supported by many researchers (Ketema et al., 2010; Sharma and Goyal, 2015). However, such resistance should be intrinsic and non-transferable; as intrinsic resistance and resistance due to mutation of chromosomal genes present a low risk of horizontal dissemination. Further, antimicrobial potential of LAB further enhances the efficacy of antimicrobial drug therapy, as it also helps in eradicating the pathogenic microorganism (Sharma et al., 2017b). In the current study, we have observed a wide range of resistance among goat Lactobacillus isolates; therefore further studies are needed to understand the resistance mechanisms on molecular basis. 
Also there is a need to put a check and ensure prudent use of antibiotics falling under intermediate susceptibility.

\section{Acknowledgements}

The authors would like to acknowledge the financial support from SERB-MoFPI (SERB/MoFPI/026/2015) and SERB-DST (SB/YS/LS-131/2014), Government of India for carrying out the research work.

\section{References}

Anas, M., Ahmed, K and Mebrouk, K., 2014. Study of the antimicrobial and probiotic effect of Lactobacillus plantarum (P6) isolated from raw goat's milk from the region of western Algeria. World Appl. Sci. J. 32(7), 1304-1310.

Ashraf, R and Shah, N.P., 2011. Antibiotic resistance of probiotic organisms and safety of probiotic dairy products. Int. Food Res. J. 18(3), 837-853.

Baines, S.D and Wilcox, M.H., 2015. Antimicrobial resistance and reduced susceptibility in Clostridium difficile: Potential consequences for induction, treatment and recurrence of $C$. difficile infection. Antibiotics 4, 267-298.

Bhuiyan, R., Shill, S., Islam, A and Chakrabortty, S., 2017. Screening of Lactobacillus spp. from raw goat milk showing probiotic activities against pathogenic bacteria. African J. Microbiol. Res. 11(15), 620-625.

Bode, L., 2006. Recent advances on structure, metabolism, and function of human milk oligosaccharides. J. Nutr. 136, $2127-$ 2130.

Bouridane, H., Sifour, M., Idoui, T., Annick, L and Thonard, P., 2016. Technological and Probiotic Traits of the Lactobacilli Isolated From Vaginal Tract of the Healthy Women for Probiotic Use. Iran J. Biotech. 14(3), e1432.

Bousmaha-Marroki, L and Marroki, A., 2015. Antibiotic susceptibility and heterogeneity in technological traits of lactobacilli isolated from Algerian goat's milk. J. Food Sci. Technol. 52(8), 47084723.

Delgado, S., Flrez, A.B and Mayo, B., 2005. Antibiotic susceptibility of Lactobacillus and Bifidobacterium species from the human gastrointestinal tract. Curr. Microbiol. 50, 202-207.

Gad, G.F.M., Abdel-Hamid, A.M and Farag, Z.S.H., 2014. Antibiotic resistance in lactic acid bacteria isolated from some pharmaceutical and dairy products. Braz. J. Microbiol. 45(1), 25-33.

Goldstein, E.J.C., Tyrrell, K.L and Citron, D.M., 2015. Lactobacillus Species: Taxonomic Complexity and Controversial Susceptibilities. Clin. Infect. Dis. 60(S2), S98-107.

Halder, D and Shyamapada, M., 2016. Antibacterial potentiality of commercially available probiotic lactobacilli and curd lactobacilli strains, alone and in combination, against human pathogenic bacteria. Translational Biomedicine, 7(2):61. doi: $\quad 10.21767 / 2172-$ 0479.100061 .

Hummel, A.S., Hertel, C., Holzapeel, W.H and Franz, C.M., 2007. Antibiotic resistances of starter and probiotic strains of lactic acid bacteria. Appl. Environ. Microbiol. 73, 730-739.

Jiang, M., Zhang, F., Wan, C., Ziong, Y., Shah, N.P., Wei, H and Tao, X., 2016. Evaluation of probiotic properties of Lactobacillus plantarum WLPL04 isolated from human breast milk. J. Dairy Sci. 99, 1-11.

Jose, N.M., Bunt, C.R and Hussain, M.A., 2015. Comparison of Microbiological and Probiotic Characteristics of Lactobacilli Isolates from Dairy Food Products and Animal Rumen Contents. Micro organisms 3, 198-212.

Karapetkov, N., Georgieva, R., Rumyan, N and Karaivanova, E., 2011. Antibiotic susceptibility of different lactic acid bacteria strains. Benef. Microbes 2, 335339. 
Ketema, B., Tetemke, $\mathrm{M}$ and Mogessie, A., 2010. Antibiotic susceptibility patterns of LAB isolated from Wakalim, a traditional Ethiopian fermented beef sausage. J. Food Safety 30, 213-223.

Marroki, A., Zúñiga, M., Kihal, M and PérezMartínez, G., 2011. Characterization of lactobacillus from Algerian goat's milk based on phenotypic, 16S rDNA sequencing and their technological properties. Braz. J. Microbiol. 42, 158171.

Munoz, M.D.C.C., Benomar, N., Lerma, L.L., Galvez, A. and Abriouel, H., 2014. Antibiotic resistance of Lactobacillus pentosus and Leuconostoc pseudomesenteroides isolated from naturally-fermented Alorena table olives throughout fermentation process. Int $\mathbf{J}$ Food Microbiol, 172, 110-118.

Nawaz, M., Wang, J., Zhou, A., Ma, C., Wu, X., Moore, J.E., Miller, B.C and Xu, J., 2011. Characterization and transfer of antibiotic resistance in lactic acid bacteria from fermented food products. Curr. Microbiol. 62, 1081-1089.

Panwar, H., Calderwood, D., Gillespie, A., Wylie, A.R., Graham, S.F., Grant, I.R., Grover, S and Green, B.D., 2016. Identification of lactic acid bacteria strains modulating incretin hormone secretion and gene expression in enteroendocrine cells. J. Funct. Foods 23, 348-358.

Pfeifer, Y., Cullik, A and Witte, W., 2010. Resistance to cephalosporins and carbapenems in Gram negative bacterial pathogens. Int. J. Med. Microbiol.300, 371-379.

Seddik, H.A., Bendali, F., Gancel, F., Fliss, I., Spano, G and Drider, D., 2017. Lactobacillus plantarum and its probiotic and food potentialities. Probiotics Antimicro. Prot. DOI: 10.1007/s12602017-9264-z.

Sharma, C., Gulati, S., Thakur, N., Singh, B.P., Gupta, S., Kaur, S., Mishra, S.K., Puniya, A.K., Gill, J.P.S and Panwar, H., 2017a. Antibiotic sensitivity pattern of indigenous lactobacilli isolated from curd and human milk samples. 3 Biotech 7(1), 53.

Sharma, C., Singh, B.P., Thakur, N., Gulati, S., Gupta, S., Mishra, S.K., Panwar, H., 2017b. Antibacterial effects of Lactobacillus isolates of curd and human milk origin against food borne and human pathogens. 3 Biotech 7(31).

Sharma, J and Goyal, A., 2015. Study on the drug resistance of probiotic strains isolated from commercial probotic products available in the local market of Agra. Eur. J. Exp. Biol. 5(2), 33-36.

Silanikove, N., Leitner, G., Merinc, $\mathrm{U}$ and Prosser, C.G., 2010. Recent advances in exploiting goat's milk: Quality, safety and production aspects. Small Ruminant Res. 89, 110-124.

Van Hoek, A.H.A.M., Mevius, D., Guerra, B., Mullany, P., Roberts, A.P and Aarts, H.J.M., 2011. Acquired antibiotic resistance genes: an overview. Front Microbiol. doi: 10.3389/fmicb.2011. 00203.

\section{How to cite this article:}

Nishchal Thakur, Chetan Sharma, Namita Rokana, Brij Pal Singh, Rohini Devidas Gulhane, Santosh Kumar Mishra, Sunil Kumar Khatkar, Anil Kumar Puniya and Harsh Panwar. 2018. In vitro Assessment of Antibiotic Resistance Pattern among Lactobacillus Strains Isolated from Goat Milk. Int.J.Curr.Microbiol.App.Sci. 7(01): 2108-2116. doi: https://doi.org/10.20546/ijcmas.2018.701.254 